\title{
Notation for chemical arrangements in alloys
}

\author{
Kengo Nishio ${ }^{*}$ and Takehide Miyazaki \\ National Institute of Advanced Industrial Science and Technology (AIST), Central 2, Umezono 1-1-1, Tsukuba, Ibaraki 305-8568, Japan
}

(Received 8 October 2019; accepted 20 April 2020; published 19 May 2020)

\begin{abstract}
Since there is no proper method to classify arrangements of atoms of different elements—chemical arrangements - the atomic structure of liquid and glassy alloys is hidden under a veil of mystery. Here we present a method to represent chemical arrangements by unique number sequences, which can be readily classified. This method paves the way for statistically studying chemical arrangements and thus provides further insight into the physics of disordered materials. As a demonstration, we apply it to uncover possible chemical arrangements responsible for preventing crystallization of a supercooled liquid alloy.
\end{abstract}

DOI: 10.1103/PhysRevResearch.2.023193

Classification of objects is essential to all branches of the humanities and sciences. In physics, the arrangements of atoms in materials are classified to better understand them and their relation to the materials' properties. Group theory provides a means of classifying the regular arrangements of atoms, and physical properties of crystals are explained in terms of their crystal structures [1]. By contrast, our knowledge of the properties of disordered materials such as liquids and glasses is much less than satisfactory. This is due to the lack of a proper method to classify the random arrangements of atoms.

The atomic arrangement can be represented as the tiling of Voronoi polyhedra using Voronoi tessellation [2-5]. Each polyhedron contains one atom and represents the geometry of the local structure composed of the central atom and its neighbors. Here the term "geometry" indicates that the positions of the atoms are concerned, but which element is assigned to each position is ignored. Recently, a method called the $p_{3}$ code has been proposed to classify the Voronoi polyhedra and their corresponding local geometrical arrangements [6-8]. This method has unveiled the universal geometrical short-range order of monatomic simple liquids and glasses [9,10].

To understand the local structure of multicomponent disordered materials, we need to know the chemical arrangement in the local structure. Here the term "chemical" indicates that, in addition to the positions of the atoms, which element is assigned to each position is concerned. One approach to characterize chemical short-range order is the chemical composition [11-13]. However, knowing only the chemical composition is not enough to establish the full picture of the chemical arrangements. We illustrate this by a simple example. The local structures shown in Figs. 1(a) and 1(b) have the same topology (octahedral structure) and the same

\footnotetext{
*k-nishio@aist.go.jp

Published by the American Physical Society under the terms of the Creative Commons Attribution 4.0 International license. Further distribution of this work must maintain attribution to the author(s) and the published article's title, journal citation, and DOI.
}

chemical composition (two red atoms and five blue atoms). However, they have different chemical arrangements. In this case, the two chemical arrangements can be distinguished by noting that the red atoms are separate in Fig. 1(a) and the red atoms are adjacent in Fig. 1(b). However, in the study of disordered materials, a myriad of chemical arrangements need to be examined, and it is practically impossible to classify them by words.

In this paper, we present compact notation for chemical arrangements so that myriad chemical arrangements can be readily classified. This is achieved by coloring each Voronoi polyhedron according to the corresponding chemical elements and generalizing the $p_{3}$ code to describe the colored Voronoi polyhedron. Its usefulness is demonstrated for a liquid alloy.

We first explain the original $p_{3}$ code in which the color of polyhedra is not considered [6-8] and then generalize it to deal with colored polyhedra. We regard a polyhedron as an object made of polygons glued together side by side and focus on its topology. In other words, the networks of the polygons are concerned, but the lengths and angles in the polyhedron are not. The polyhedron is considered to be identical to its mirror image. As described later, the $p_{3}$ code is a method to encode the topology of a polyhedron as a unique number sequence called a $p_{3}$ codeword so that the original polyhedron can be recovered from $p_{3}$.

To encode a polyhedron, we first choose a polygon and one of its sides as a seed. Different seeds yield different codewords. The mirror-image polyhedron also yields different codewords. As described later, each $p_{3}$ has a (a)

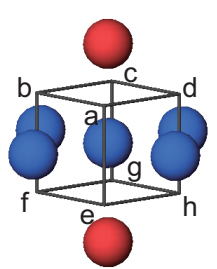

(b)

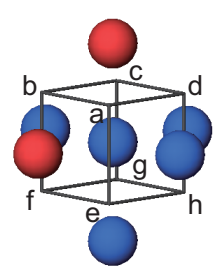

FIG. 1. Local structures which have the same topology and the same chemical composition but different arrangements of the red and blue atoms. 
lexicographical order $\operatorname{Lex}\left(p_{3}\right)$ and the one with the smallest $\operatorname{Lex}\left(p_{3}\right)$ is defined as the unique codeword. In general, $p_{3}$ consists of $p s_{2}$ and $s p$ codewords and is expressed as $p_{3}=$ $p s_{2} ; s p$, where the semicolon is a separator. The $p s_{2}$ codeword is a number sequence $p_{2}(1) p_{2}(2) p_{2}(3) \cdots p_{2}(P)$, where $p_{2}(i)$ is the number of sides of the polygon $i$ and $P$ is the number of polygons of the polyhedron. Generating $p s_{2}$ is assigning identification numbers (IDs) to the polygons. For this purpose, we also assign $i_{j}$ to the $j$ th side of the polygon $i$. The side ID $i_{j}$ represents an integer: $i_{j}=j+\sum_{x=1}^{i-1} p_{2}(x)$. In the course of encoding, IDs are assigned to sides step by step. When a side to which an ID is already assigned is glued to a side to which an ID is not assigned yet, the former is called a dangling side. For a given seed, $p s_{2}$ is obtained as follows. (1) The seed polygon is polygon 1 and IDs $\left(1_{1}, 1_{2}, 1_{3}, \ldots, 1_{p_{2}(1)}\right)$ are assigned to its sides in the clockwise direction from the seed side. (2) The polygon glued to the dangling side with the smallest ID is the next polygon $i$ and IDs $\left(i_{1}, i_{2}, i_{3}, \ldots, i_{p_{2}(i)}\right)$ are assigned to its sides in the clockwise direction from the side glued to the smallest-ID dangling side. (3) Repeat procedure 2 until IDs are assigned to all the polygons and their sides.

In most cases, $p_{3}$ does not contain $s p$ and the original polyhedron can be recovered from $p_{3}=p s_{2}$ using the decoding algorithm described later. When the original polyhedron cannot be recovered from $p s_{2}, p_{3}$ contains $s p$ and the original polyhedron can be recovered from $p_{3}=p s_{2} ; s p$. Here $s p$ is a number sequence $y(1) x(1) y(2) x(2) y(3) x(3) \cdots y(N) x(N)$. The pair $y(i) x(i)$ indicates that the side $y(i)$ is glued to the side $x(i) . N$ is the number of $y x$ pairs. See Refs. [6-8] for how to generate $s p$.

In the decoding process, we glue the polygons side by side. In this process, a side that is not glued to other side is also called a dangling side. The $p_{3}$ codeword instructs how to assemble the polygons as follows. (1) The polygon $\alpha$ is a $p_{2}(\alpha)$-gon $(1 \leqslant \alpha \leqslant P)$ and IDs $\left(\alpha_{1}, \alpha_{2}, \alpha_{3}, \ldots, \alpha_{p_{2}(\alpha)}\right)$ are assigned to its sides in the clockwise direction. (2) The polygon 1 is the partial polyhedron 1. (3) Make the next partial polyhedron $i$ as follows. Glue the side $i_{1}$ of the polygon $i$ to the smallest-ID dangling side of the partial polyhedron $i-1$. When the side $y(\beta)(1 \leqslant \beta \leqslant N)$ is a side of the polygon $i$, glue the sides $y(\beta)$ and $x(\beta)$ together. When three corners meet at a junction of two dangling sides, glue the dangling sides together. Note that each corner of a polyhedron is assumed to meet exactly two other corners. The Voronoi polyhedra of disordered structures satisfy this assumption. (4) Repeat procedure 3 until all the polygons are assembled.

The lexicographical order $\operatorname{Lex}\left(p_{3}\right)$ is the concatenation of $\operatorname{Lex}\left(p s_{2}\right)$ and $\operatorname{Lex}(s p)$. Here $\operatorname{Lex}\left(p s_{2}\right)$ is a $P$-digit base$W$ number $\left(p s_{2}\right)_{W}=\left(p_{2}(1) p_{2}(2) p_{2}(3) \cdots p_{2}(P)\right)_{W}$, where $W$ is any sufficiently large number, e.g., $W=6 P$. Similarly, $\operatorname{Lex}(s p)=(s p)_{W}$ is a $2 N$-digit base- $W$ number.

A polyhedron represented by $p_{3}$ is called a $p_{3}$ polyhedron. The local structure corresponding to a $p_{3}$ Voronoi polyhedron is represented by @ $p_{3}$. The topology of a local structure is encoded as @ $p_{3}$. However, it does not contain information on the chemical arrangement in the local structure.

Next we generalize the original $p_{3}$ code to describe the chemical arrangements. To encode the chemical arrangement

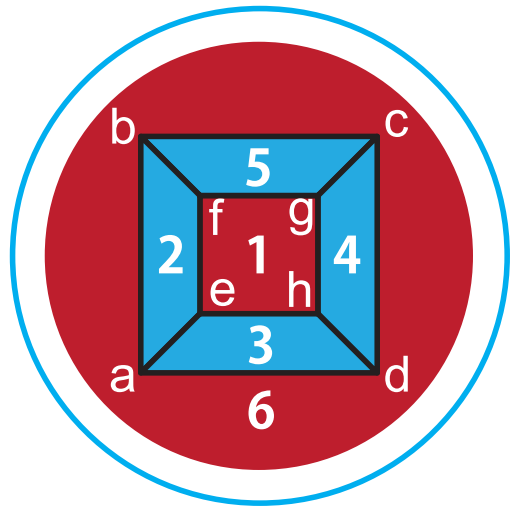

FIG. 2. Circled Schlegel diagram of the colored Voronoi polyhedron of the local structure shown in Fig. 1(a). The color of the circle, blue, represents the color of the interior of the Voronoi polyhedron. The polygon corresponding to the red (blue) neighbor is colored in red (blue). The number in each polygon indicates the polygon ID which is assigned when the polygon fehg and the side $f e$ are chosen as a seed. Note that the outside of the polygon abcd of the Schlegel diagram corresponds to the inside of the polygon abcd of the polyhedron. The clockwise direction around the polygon $a b c d$ of the Schlegel diagram corresponds to the clockwise direction around the corresponding polygon of the polyhedron. For the other polygons, the counterclockwise direction of the Schlegel diagram corresponds to clockwise direction of the polyhedron.

in a local structure, we first assign color to each atom in that local structure according to its atomic elements. We then color the interior of the Voronoi polyhedron according to the color of the central atom. We also color each polygon of the Voronoi polyhedron according to the color of the corresponding atom. As an example, the colored Voronoi polyhedron of the local structure shown in Fig. 1(a) is illustrated in Fig. 2 using a Schlegel diagram.

The chemical arrangement is now represented as the corresponding colored Voronoi polyhedron. To encode it, we introduce color IDs. Let us assume that the color IDs of red and blue are $r$ and $b$, respectively, and that $0 \leqslant r<b<W$. Note that, although the definition of the color IDs is arbitrary, a unique codeword is assigned to each chemical arrangement for a given definition. We then define the color sequence codeword $c s$ as follows:

$$
c s=c(0) c(1) c(2) \cdots c(P) .
$$

Here $c(0)$ is the color ID of the interior. If the interior is red (blue), then $c(0)=r[c(0)=b]$. For $1 \leqslant i \leqslant P, c(i)$ is the color ID of the polygon $i$. If the polygon $i$ is red (blue), then $c(i)=r[c(i)=b]$. We then generalize $p_{3}$ as

$$
p_{3}=p s_{2} ; s p ; c s \text {. }
$$

When $p_{3}$ does not contain $s p$, we express $p_{3}$ as

$$
p_{3}=p s_{2} ; c s,
$$

where ";;" is contracted to ";". We also generalize Lex $\left(p_{3}\right)$ as the concatenation of $\operatorname{Lex}\left(p s_{2}\right)$, $\operatorname{Lex}(s p)$, and $\operatorname{Lex}(c s)$. Here, $\operatorname{Lex}(c s)=(c s)_{W}$ is a $(P+1)$-digit base- $W$ number. The 


$$
@ 4^{6} ; b r b^{4} r=@ 44^{4} 4
$$

FIG. 3. Brief representation of @ $p_{3}$ by using color. The color of the symbol@ represents the color of the central atom. The color of each $p_{2}(i)$ represents the color of the $i$ th neighbor of the central atom.

codeword with the smallest $\operatorname{Lex}\left(p s_{2} ; s p ; c s\right)$ is defined as the unique codeword of the colored polyhedron.

For a given seed, the colored polyhedron is encoded as follows. We first ignore the color of the polyhedron and generate the original $p s_{2} ; s p$ codeword. As a result, IDs are assigned to all the polygons. We then determine $c(i)(1 \leqslant i \leqslant$ $P$ ) from the color of the polygon $i$. We also determine $c(0)$ from the color of the interior. The colored polyhedron can be recovered from its $p s_{2} ; s p ; c s$ as follows. We first make a plain polyhedron from $p s_{2} ; s p$ and then color the polyhedron according to $c s$.

We explain how to encode the colored Voronoi polyhedron shown in Fig. 2 as an example. Since the central atom of the polyhedron is blue, $c(0)=b$. When we generate $p s_{2}$ with choosing the polygon $f e h g$ and the side $f e$ as a seed, IDs 1 , $2,3,4,5$, and 6 are assigned to the polygons $f e h g$ (red), ef $b a$ (blue), head (blue), ghdc (blue), fgcb (blue), and abcd (red), respectively. Thus, $p s_{2}=444444$ and $c s=b r b b b b r$. We abbreviate these as $p s_{2}=4^{6}$ and $c s=b r b^{4} r$, respectively. If we ignore color, the polyhedron can be recovered from $p s_{2}=$ $4^{6}$. Therefore, $p_{3}$ does not contain $s p$, and $p_{3}=4^{6} ; b r b^{4} r$. By choosing different seeds, two additional different $p_{3} \mathrm{~s}$, $4^{6} ; b^{2}(r b)^{2} b$ and $4^{6} ; b^{3}(r b)^{2}$, are obtained. The lexicographically smallest $4^{6} ; b r b^{4} r$ is the unique codeword. Since its Voronoi polyhedron is $4^{6} ; b r b^{4} r$, the local structure shown in Fig. 1(a) is represented by @ $4^{6} ; b r b^{4} r$. This codeword differs from @ $4^{6} ; b r^{2} b^{4}$ of the local structure shown in Fig. 1(b), exemplifying that the generalized $p_{3}$ code can distinguish different chemical arrangements. By coloring the codeword, the chemical arrangement can be expressed more briefly as illustrated in Fig. 3.

Note that the present method can be generalized to classify chemical arrangements in complexes of local structures in a similar way that the original $p_{3}$ code is generalized to the $p_{4}$ code [6-8].

To demonstrate the usefulness of our notation, we investigate chemical arrangements in a popular model of supercooled liquid alloy proposed by Wahnström $[14,15]$. This (a)

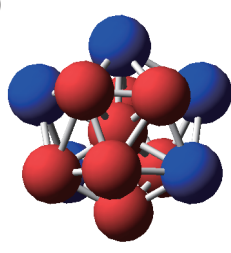

(a) $5^{5} 555^{4} 5$ (b)

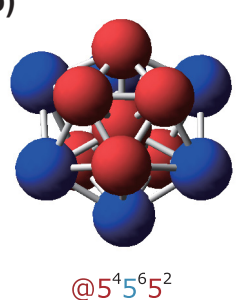

(c)

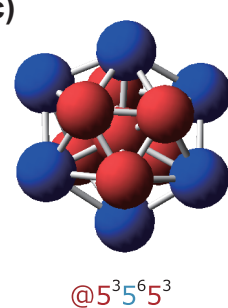

FIG. 4. Chemically different $S$-centered icosahedral structures found in the Wahnström liquid. Here $S$ and $L$ atoms are colored red and blue, respectively. (a) Most favored chemical arrangements. (b) and (c) Crystalline chemical arrangements.

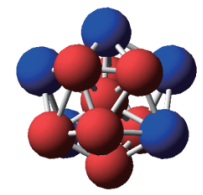

(a) $5^{5} 555^{4} 5$

$(3.5 \%,-6.89)$

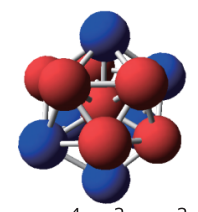

(a) $5^{4}\left(5^{2} 55\right)^{2}$

$(3.1 \%,-6.89)$

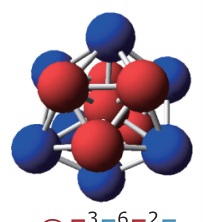

(a) $5^{3} 5^{6} 5^{2} 5$

$(2.9 \%,-7.02)$

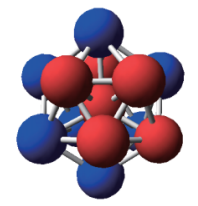

(a) $5^{4} 5^{6} 55$

$(2.7 \%,-6.99)$

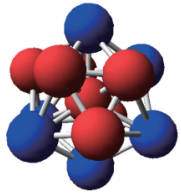

(a) $5^{3} 555^{4} 555$

$(3.3 \%,-6.91)$

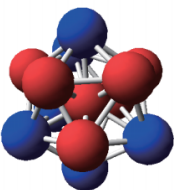

(a) $5^{3}(55)^{2} 5^{2} 55^{2}$

$(3.0 \%,-6.90)$

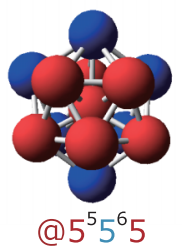

$(2.9 \%,-6.98)$

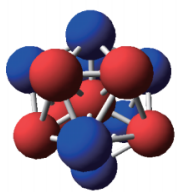

(a) $5^{2}(555)^{2} 55^{3}$

$(2.6 \%,-6.91)$

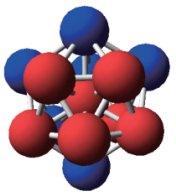

(a) $5^{5} 555^{5}$

$(3.2 \%,-6.88)$

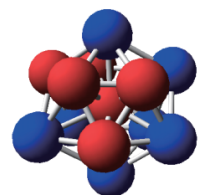

(a) $5^{3} 555^{4} 55^{2}$

$(3.0 \%,-6.91)$

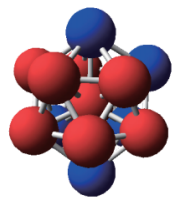

(a) $5^{5}(555)^{2} 5$

$(2.9 \%,-6.85)$

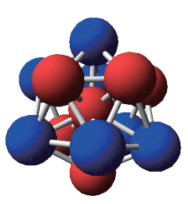

(a) $5^{2} 555^{6} 5^{2}$

$(2.6 \%,-7.03)$
FIG. 5. Noncrystalline $S$-centered icosahedra that are energetically less favored but occur more frequently than the crystalline ones. The frequency of occurrence and the average energy of each structure are also given.

model consists of 5000 small $(S)$ and 5000 large $(L)$ atoms interacting via Lennard-Jones (LJ) potentials. The interaction parameters are $\sigma_{S S}=\sigma_{S L} / 1.1=\sigma_{L} / 1.2, \epsilon_{S S}=\epsilon_{S L}=\epsilon_{L}$, and $m_{S}=m_{L} / 2$. Hereafter, the $L$-atom $L J$ reduced units are used. We perform isothermal-isobaric molecular dynamics simulations at a temperature of 0.6853 and a pressure of 10 [9,16-20]. A trajectory of $6.0 \times 10^{5} \mathrm{LJ}$ time units is sampled and divided into three parts to evaluate the statistical uncertainty. The local structures in the inherent structure is analyzed every $100 \mathrm{LJ}$ time units. The local structures are defined by radical Voronoi tessellation [21]. The Wahnström liquid is characterized by the predominance of $S$-centered @ $5^{12}$ (icosahedral structure) [15]. Our original $p_{3}$ analysis confirms it and shows that $(24.3 \pm 0.4) \%$ of the $S$-centered local structures adopt this topology. We therefore focus on the chemical arrangements in the $S$-centered icosahedra. Atomic coordinates and generalized $p_{3}$ codewords of inherent structures at $2.0 \times 10^{5}, 4.0 \times 10^{5}$, and $6.0 \times 10^{5} \mathrm{LJ}$ time units are provided in the Supplemental Material [22]. 
By using the generalized $p_{3}$ analysis, we uncover a total of 82 chemically different types of $S$-centered icosahedra. In particular, the most favored chemical arrangement is found to be @ $5^{12} ; s^{6} l s l^{4} s$ [Fig. 4(a)] and $(3.5 \pm 0.1) \%$ of the $S$ centered icosahedra adopt this chemical arrangement. Here we assume that the color IDs of $S$ and $L$ atoms are $s$ and $l$, respectively, and that $0 \leqslant s<l<W$. The chemical arrangements found in the crystalline phase of the Wahnström system [23,24], @ $5^{12} ; s^{5} l^{6} s^{2}$ [Fig. 4(b)] and @ $5^{12} ; s^{4} l^{6} s^{3}$ [Fig. 4(c)], are less favored. Their proportions are $(2.2 \pm 0.2) \%$ and $(0.8 \pm 0.1) \%$, respectively.

To elucidate why the noncrystalline local structure is more favored than the crystalline ones, we calculate the average energy per atom of the @ $p_{3}$ local structures [9]. The average energy for the most favored noncrystalline $@ 5^{12} ; s^{6} l s l^{4} s$ is $-6.89 \pm 0.01$, which is higher than those for the crystalline @ $5^{12} ; s^{5} l^{6} s^{2}(-7.07 \pm 0.03)$ and @ $5^{12} ; s^{4} l^{6} s^{3}$ $(-7.06 \pm 0.02)$. Thus, the noncrystalline local structure is found to be entropically more favored than the crystalline ones. In addition, it is found that a total of 12 chemical arrangements, @ $5^{12} ; s^{6} l s l^{4} s$, @ $5^{12} ; s^{4} l s l^{4} s l s$, @ $5^{12} ; s^{6} l s l^{5}$, $@ 5^{12} ; s^{5}\left(l^{2} s l\right)^{2}, \quad @ 5^{12} ; s^{4}(l s)^{2} l^{2} s l^{2}, \quad @ 5^{12} ; s^{4} l s l^{4} s l^{2}$, $@ 5^{12} ; s^{4} l^{6} s^{2} l, @ 5^{12} ; s^{6} l^{6} s, @ 5^{12} ; s^{6}(l s l)^{2} l, @ 5^{12} ; s^{5} l^{6} s l$, $@ 5^{12} ; s^{3}(l s l)^{2} s l^{3}$, and @ $5^{12} ; s^{3} l s l^{6} s^{2}$, are energetically less favored but occur more frequently than the crystalline ones (Fig. 5).

In the past, it was believed that icosahedral local structures prevented supercooled liquids from crystallization [25]. However, it was shown that the Wahnström liquid crystallizes into a Laves phase which contains $S$-centered icosahedra $[23,24]$. Since $S$-centered icosahedra can be regarded as fragments of the crystal, supercooling of the liquid cannot be explained if the chemical arrangements are ignored. Our analysis reveals the predominance of the entropy-driven noncrystalline chemical arrangements in $S$-centered icosahedra. This explains why the supercooled liquid alloy is stable despite the fact that it contains many $S$-centered icosahedra.

In conclusion, we have presented compact notation for chemical arrangements. This method paves the way for statistically studying chemical arrangements. As a demonstration, we have applied it to uncover possible chemical arrangements responsible for preventing crystallization of a supercooled liquid alloy. Our compact notation for chemical arrangements will be indispensable for advancing the physics of disordered materials as the compact notation for numbers, which allows us to handle numbers efficiently, is indispensable for the progress of science and technology [26].
[1] M. S. Dresselhaus, G. Dresselhaus, and A. Jorio, Group Theory, 4th ed. (Springer, Berlin, 2008).

[2] J. D. Bernal, Nature (London) 183, 141 (1959).

[3] J. L. Finney, Philos. Mag. 93, 3940 (2013).

[4] F. Yonezawa, Solid State Phys. 45, 179 (1991).

[5] Y. Cheng and E. Ma, Prog. Mater. Sci. 56, 379 (2011).

[6] K. Nishio and T. Miyazaki, Sci. Rep. 6, 23455 (2016).

[7] K. Nishio and T. Miyazaki, Sci. Rep. 7, 40269 (2017).

[8] K. Nishio and T. Miyazaki, in Nanoinformatics, edited by I. Tanaka (Springer Singapore, Singapore, 2018), pp. 97-130.

[9] K. Nishio, A. K. A. Lu, and T. Miyazaki, Phys. Rev. E 99, 022121 (2019).

[10] K. Nishio, A. K. A. Lu, and T. Miyazaki, Phys. Rev. Res. 1, 012013(R) (2019).

[11] J. M. Cowley, Phys. Rev. 77, 669 (1950).

[12] G. S. Cargill and F. Spaepen, J. Non-Cryst. Solids 43, 91 (1981).

[13] H. Tian, C. Zhang, L. Wang, J. Zhao, C. Dong, B. Wen, and Q. Wang, J. Appl. Phys. 109, 123520 (2011).

[14] G. Wahnström, Phys. Rev. A 44, 3752 (1991).
[15] D. Coslovich and G. Pastore, J. Chem. Phys. 127, 124504 (2007).

[16] S. Nosé, J. Chem. Phys. 81, 511 (1984).

[17] S. D. Bond, B. J. Leimkuhler, and B. B. Laird, J. Comput. Phys. 151, 114 (1999).

[18] S. Nosé, J. Phys. Soc. Jpn. 70, 75 (2001).

[19] H. C. Andersen, J. Chem. Phys. 72, 2384 (1980).

[20] S. Nosé, Mol. Phys. 52, 245 (1984).

[21] B. Gellatly and J. Finney, J. Non-Cryst. Solids 50, 313 (1982).

[22] See Supplemental Material at http://link.aps.org/supplemental/ 10.1103/PhysRevResearch.2.023193 for atomic coordinates and generalized $p_{3}$ codewords of inherent structures at $2.0 \times$ $10^{5}, 4.0 \times 10^{5}$, and $6.0 \times 10^{5} \mathrm{LJ}$ time units.

[23] U. R. Pedersen, N. P. Bailey, J. C. Dyre, and T. B. Schrder, arXiv:0706.0813.

[24] U. R. Pedersen, T. B. Schrøder, J. C. Dyre, and P. Harrowell, Phys. Rev. Lett. 104, 105701 (2010).

[25] F. C. Frank, Proc. R. Soc. London Ser. A 215, 43 (1952).

[26] T. Dantzig and J. Mazur, Number: The Language of Science, 4th ed. (Penguin Group, New York, 2007). 